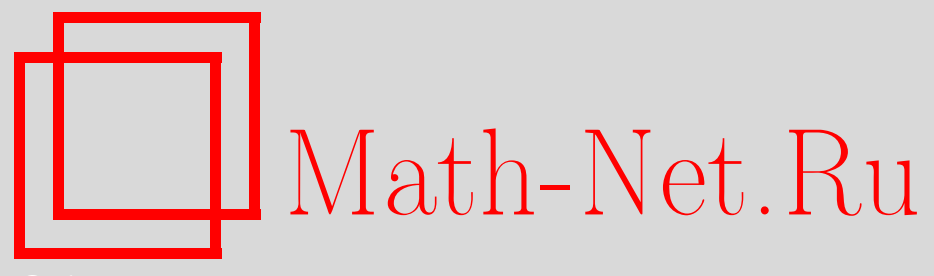

Н. В. Илюшечкин, О соотношениях между слагаемыми дискриминанта симметрической матрицы, Матем. заметки, 2013, том 93, выпуск 2, 310-312

DOI: https://doi.org/10.4213/mzm7828

Использование Общероссийского математического портала Math-Net.Ru подразумевает, что вы прочитали и согласны с пользовательским соглашением http://www . mathnet.ru/rus/agreement

Параметры загрузки:

IP : 54.172 .240 .79

26 апреля 2023 г., 14:56:59

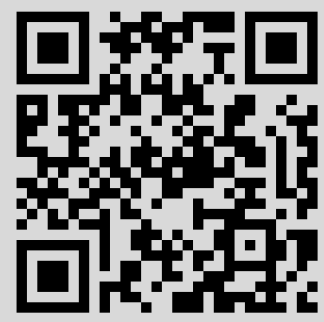




\section{О соотношениях между слагаемыми дискриминанта симметрической матрицы}

\section{Н. В. Илюшечкин}

Пусть $\operatorname{Sym}(n)$ - пространство вещественных симметрических матриц порядка $n$, матрица $X \in \operatorname{Sym}(n)$, a $\operatorname{Dis}(X)$ - дискриминант ее характеристического многочлена, который для простоты называют дискриминантом матрицы $X$. Как показали Вигнер и фон Нейман, уравнение $\operatorname{Dis}(X)=0$ выделяет в пространстве $\operatorname{Sym}(n)$ алгебраическое многообразие $\mathscr{M}$ коразмерности 2. Связь этого факта с квантовой механикой и его доказательство приводятся в [1]. Доказательство основано на нестандартной параметризации матриц из $\operatorname{Sym}(n)$ посредством их собственных векторов и собственных значений. Там же дается другое обоснование коразмерности в случае $n=3$. Именно, многообразие $\mathscr{M}$ рассматривается как множество решений системы десяти полиномиальных (однородных третьей степени) уравнений относительно элементов матрицы $X$. Кроме того, в [1] исследуется структура многообразия $\mathscr{M}$ при $n=3$ и вопрос о его неприводимых составляющих.

Здесь также исследуется многообразие $\mathscr{M}$ симметрических матриц с кратными собственными значениями при $n=3$. Цель работы - описать это многообразие как множество решений системы полиномиальных уравнений и, кроме того, дать некоторые алгебраические соотношения между этими уравнениями, чего нет в [1]. Найденные ниже соотношения позволят сократить число задающих $\mathscr{M}$ уравнений до четырех. Проводимое ниже исследование основано на представлении дискриминанта в виде суммы квадратов некоторых многочленов от элементов матрицы $X$, найденном в [2]. В [3] это представление было распространено на нормальные матрицы, в [4] дан геометрический вывод.

Опишем упомянутое представление. Пусть матрица $X=\left(x_{i j}\right)$, причем $x_{i j}=x_{j i}$. Обозначим ее квадрат через $X^{2}=\left(x_{i j}^{(2)}\right)$ и рассмотрим матрицу

$$
X_{!}=\left(\begin{array}{ccc}
1 & x_{11} & x_{11}^{(2)} \\
1 & x_{22} & x_{22}^{(2)} \\
1 & x_{33} & x_{33}^{(2)} \\
0 & \sqrt{2} x_{12} & \sqrt{2} x_{12}^{(2)} \\
0 & \sqrt{2} x_{13} & \sqrt{2} x_{13}^{(2)} \\
0 & \sqrt{2} x_{23} & \sqrt{2} x_{23}^{(2)}
\end{array}\right) .
$$

Как показано в [3], дискриминант $\operatorname{Dis}(X)$ равен сумме квадратов всех максимальных миноров матрицы $X$ ! Займемся сначала подсчетом числа различных ее миноров. Прежде

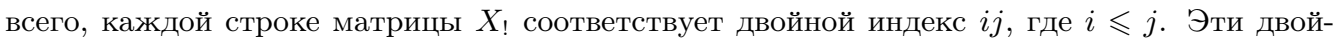
ные индексы расположим в порядке $11,22,33,12,13,23$. Минор, образованный строками с двойными индексами $i_{1} j_{1}, i_{2} j_{2}, i_{3} j_{3}$, обозначим через $\left\langle i_{1} j_{1}, i_{2} j_{2}, i_{3} j_{3}\right\rangle$. Очевидно, число всех таких миноров равно $C_{6}^{3}=20$. При этом $\langle 12,13,23\rangle=0$. Кроме того, если $i_{2}<j_{2}$ и $i_{3}<j_{3}$, то, очевидно, $\left\langle 11, i_{2} j_{2}, i_{3} j_{3}\right\rangle=\left\langle 22, i_{2} j_{2}, i_{3} j_{3}\right\rangle=\left\langle 33, i_{2} j_{2}, i_{3} j_{3}\right\rangle$, т.е. имеется три тройки совпадающих миноров. Наконец, непосредственно видно, что

$$
\begin{aligned}
& \langle i i, 12,13\rangle=\sqrt{2}\langle 22,33,23\rangle=2 x_{12} x_{13}\left(x_{33}-x_{22}\right)-2 x_{23}\left(x_{13}^{2}-x_{12}^{2}\right), \\
& \langle i i, 12,23\rangle=\sqrt{2}\langle 11,33,13\rangle=2 x_{12} x_{23}\left(x_{33}-x_{11}\right)-2 x_{13}\left(x_{23}^{2}-x_{12}^{2}\right), \\
& \langle i i, 13,23\rangle=\sqrt{2}\langle 11,22,12\rangle=2 x_{13} x_{23}\left(x_{22}-x_{11}\right)-2 x_{12}\left(x_{23}^{2}-x_{13}^{2}\right) .
\end{aligned}
$$

DOI: $10.4213 / \operatorname{mzm} 7828$ 
Таким образом, имеется всего 10 различных миноров, причем можно условиться выбирать их так, чтобы по крайней мере двум строкам любого из них соответствовали диагональные индексы вида $і$. Дискриминант можно представить в виде суммы десяти квадратов:

$$
\begin{aligned}
\operatorname{Dis}(X)=\langle & 11,22,33\rangle^{2}+7\langle 11,22,12\rangle^{2}+7\langle 11,33,13\rangle^{2}+7\langle 22,33,23\rangle^{2} \\
& +\langle 11,22,13\rangle^{2}+\langle 11,33,23\rangle^{2}+\langle 22,33,12\rangle^{2} \\
& +\langle 11,22,23\rangle^{2}+\langle 11,33,12\rangle^{2}+\langle 22,33,13\rangle^{2} .
\end{aligned}
$$

Оказывается, десять приведенных только что кубических многочленов связаны между собой восемью уравнениями первой и второй степени. Переходим к выводу этих уравнений. Прежде всего, если в матрице $X$ ! продублировать первый столбец и рассмотреть равные нулю миноры четвертого порядка, то легко найти три линейных соотношения, которые позволяют выразить все миноры вида $\langle i i, j j, i j\rangle$ через шесть миноров вида $\langle i i, j j, k l\rangle$, где $k<l$ и один из индексов $k$ или $l$ отличен от $i$ и $j$ :

$$
\begin{aligned}
& \langle 11,22,12\rangle=\langle 11,33,12\rangle-\langle 22,33,12\rangle, \\
& \langle 11,33,13\rangle=\langle 11,22,13\rangle+\langle 22,33,13\rangle, \\
& \langle 22,33,23\rangle=\langle 11,33,23\rangle-\langle 11,22,23\rangle .
\end{aligned}
$$

Как мы скоро увидим, в силу соотношений (1) миноры $\langle i i, j j, i j\rangle$ можно не привлекать к исследованию многообразия $\mathscr{M}$. Для оставшихся миноров пора упростить обозначения. Именно, положим $f_{0}=\langle 11,22,33\rangle$,

$$
\begin{array}{lll}
f_{1}=2^{-1 / 2}\langle 11,22,13\rangle, & f_{2}=2^{-1 / 2}\langle 22,33,12\rangle, & f_{3}=-2^{-1 / 2}\langle 11,33,23\rangle, \\
f_{4}=-2^{-1 / 2}\langle 22,33,13\rangle, & f_{5}=2^{-1 / 2}\langle 11,33,12\rangle, & f_{6}=-2^{-1 / 2}\langle 11,22,23\rangle .
\end{array}
$$

Это кубические формы с целыми коэффициентами. В частности,

$$
\begin{aligned}
& f_{0}=\left(x_{22}-x_{11}\right)\left(x_{33}-x_{11}\right)\left(x_{33}-x_{22}\right)-\left(x_{22}-x_{11}\right) x_{12}^{2}+\left(x_{33}-x_{11}\right) x_{13}^{2}-\left(x_{33}-x_{22}\right) x_{23}^{2}, \\
& f_{1}=x_{13}\left(x_{22}-x_{11}\right)\left(x_{33}-x_{22}\right)+x_{12} x_{23}\left(x_{22}-x_{11}\right)-x_{13}\left(x_{23}^{2}-x_{13}^{2}\right) .
\end{aligned}
$$

Остальные пять многочленов могут быть получены из $f_{1}$ перестановками индексов у переменных (о перестановках индексов см. [2] и [3]).

Что же касается дискриминанта, то

$$
\operatorname{Dis}(X)=f_{0}^{2}+\sum_{i=1}^{3}\left(16 f_{i}^{2}-28 f_{i} f_{i+3}+16 f_{i+3}^{2}\right) .
$$

Квадратичная форма $16 a^{2}-28 a b+16 b^{2}$ положительно определена, и ее можно бесконечным числом способов представить как сумму двух квадратов. Таким образом, дискриминант представим в виде суммы семи квадратов, причем многообразие $\mathscr{M}$ задается как пересечение множеств нулей семи кубических форм $f_{0}=f_{1}=\cdots=f_{6}=0$.

В частности, из тождества $16 a^{2}-28 a b+16 b^{2}=(a+b)^{2}+15(a-b)^{2}$ следует представление дискриминанта как суммы семи квадратов, данное в [5].

Теорема 1. Семъ кубических форм $f_{0}, \ldots, f_{6}$, задающих многообразие $\mathscr{M}$, удовлетворяют пяти однородным квадратичным соотношениям. Из этих соотношений следует, что $\mathscr{M}$ можно задать любой из систем четырех уравнений

$$
f_{0}=f_{1}=f_{2}=f_{3}=0 \quad \text { или } \quad f_{0}=f_{4}=f_{5}=f_{6}=0 .
$$


ДокаЗАТЕЛЬСтво. Формы $f_{0}, \ldots, f_{6}$ являются максимальными минорами матрицы $X_{!}$, вследствие чего удовлетворяют соотношениям Плюккера [6]. Напомним, что каждой строке матицы $X$ ! соответствует двойной индекс и эти индексы расположены в порядке 11,22 , $33,12,13,23$ (от меньших к большим). Применительно к рассматриваемому случаю соотношения Плюккера строятся следующим образом. Если $A, B, C, \ldots$ - двойные индексы, причем $A<B<C<D, E<F$, то

$$
\langle A, B, C\rangle\langle D, E, F\rangle-\langle A, B, D\rangle\langle C, E, F\rangle+\langle A, C, D\rangle\langle B, E, F\rangle-\langle B, C, D\rangle\langle A, E, F\rangle=0 .
$$

Возьмем в (3) $A=11, B=22, C=12, D=13, E=33, F=23$. Тогда

$$
\begin{aligned}
& \langle 11,22,12\rangle\langle 13,33,23\rangle-\langle 11,22,13\rangle\langle 12,33,23\rangle \\
& \quad+\langle 11,12,13\rangle\langle 22,33,23\rangle-\langle 22,12,13\rangle\langle 11,33,23\rangle=0 .
\end{aligned}
$$

Выражая все через многочлены $f_{i}$ и используя перестановки индексов, а именно циклы, отсюда можно получить три соотношения второй степени. Из этих соотношений независимы лишь любые два, так как сумма левых частей всех их тождественно равна нулю. Приводим два из них:

$$
\left\{\begin{array}{l}
f_{1}^{2}+f_{6}^{2}-f_{2}^{2}-f_{5}^{2}-f_{1} f_{4}+2 f_{2} f_{5}-f_{3} f_{6}=0 \\
f_{3}^{2}+f_{5}^{2}-f_{1}^{2}-f_{4}^{2}+2 f_{1} f_{4}-f_{2} f_{5}-f_{3} f_{6}=0 .
\end{array}\right.
$$

Возьмем в (3) $A=11, B=22, C=33, D=12, E=11, F=13$. Тогда

$$
\langle 11,22,33\rangle\langle 12,11,13\rangle-\langle 11,22,12\rangle\langle 33,11,13\rangle+\langle 11,33,12\rangle\langle 22,11,13\rangle=0 .
$$

Отсюда можно получить еще три различных соотношения на формы $f_{i}$ :

$$
\left\{\begin{array}{l}
f_{0}\left(f_{1}-f_{4}\right)-f_{2} f_{3}+f_{3} f_{5}-f_{5} f_{6}=0, \\
f_{0}\left(f_{2}-f_{5}\right)-f_{1} f_{3}+f_{1} f_{6}-f_{4} f_{6}=0, \\
f_{0}\left(f_{3}-f_{6}\right)-f_{1} f_{2}+f_{2} f_{4}-f_{4} f_{5}=0 .
\end{array}\right.
$$

Из соотношений (4) и (5) легко следует, что многообразие $\mathscr{M}$ можно задать любой из систем (2).

Автор благодарен В. И. Арнольду за постановку задачи.

\section{СПИСОК ЦИТИРОВАННОЙ ЛИТЕРАТУРЫ}

[1] М. Дана, Х.Д. Икрамов, Численные методы и вопросы организации вычислений. XVIII, Зап. научн. сем. ПОМИ, 323, ПОМИ, СПб., 2005, 34-46. [2] Н. В. Илюшечкин, УМН, 40:1(241) (1985), 201-202. [3] Н. В. Илюшечкин, Матем. заметки, 51:3 (1992), 16-23. [4] Н. В. Илюшечкин inbook Исследования по линейным операторам и теории функций. 31, Зап. научн. сем. ПОМИ, 303, ПОМИ, СПб., 2003, 119-144. [5] Р. A. Parrilo, Math. Program, 96:2, Ser. В (2003), 293-320. [6] М. М. Постников, Лекиии по геометрии. Семестр II. Линейная алгебра, Наука, М., 1986.

Н. В. Илюшечкин

Поступило

ОАО Концерн "Моринформсистема - Агат", 06.04.2009

г. Москва

E-mail: ilyush55@mail.ru 